(C) 2006 American Chemical Society, Organometallics, Bleeke om058058f Supporting Info Page 81

Table 3. Bond lengths $[\AA]$ and angles $\left[{ }^{\circ}\right]$ for jb14704.

\begin{tabular}{|c|c|}
\hline $\mathrm{Rh}(1)-\mathrm{C}(4)$ & $2.085(3)$ \\
\hline $\operatorname{Rh}(1)-C(1)$ & $2.107(3)$ \\
\hline $\operatorname{Rh}(1)-P(1)$ & $2.2593(9)$ \\
\hline $\mathrm{Rh}(1)-\mathrm{P}(2)$ & $2.3141(9)$ \\
\hline $\mathrm{Rh}(1)-\mathrm{S}(1)$ & $2.3906(8)$ \\
\hline$R h(1)-S(1) \# 1$ & $2.4664(7)$ \\
\hline$S(1)-C(4)$ & $1.782(4)$ \\
\hline $\mathrm{S}(1)-\mathrm{Rh}(1) \# 1$ & $2.4664(7)$ \\
\hline$P(1)-C(11)$ & $1.814(4)$ \\
\hline$P(1)-C(12)$ & $1.822(4)$ \\
\hline$P(1)-C(13)$ & $1.824(5)$ \\
\hline$P(2)-C(22)$ & $1.816(5)$ \\
\hline$P(2)-C(21)$ & $1.817(4)$ \\
\hline$P(2)-C(23)$ & $1.818(5)$ \\
\hline$C(1)-C(2)$ & $1.495(6)$ \\
\hline $\mathrm{C}(1)-\mathrm{H}(1 \mathrm{~A})$ & $0.90(3)$ \\
\hline$C(1)-H(1 B)$ & $0.89(3)$ \\
\hline$C(2)-C(3)$ & $1.329(5)$ \\
\hline$C(2)-C(5)$ & $1.498(5)$ \\
\hline$C(3)-C(4)$ & $1.492(5)$ \\
\hline$C(3)-C(6)$ & $1.500(6)$ \\
\hline $\mathrm{C}(4)-\mathrm{H}(4)$ & $0.98(4)$ \\
\hline$C(5)-H(5 A)$ & $0.93(3)$ \\
\hline $\mathrm{C}(5)-\mathrm{H}(5 \mathrm{~B})$ & $0.93(3)$ \\
\hline$C(5)-H(5 C)$ & $0.93(3)$ \\
\hline$C(6)-H(6 A)$ & $0.96(2)$ \\
\hline $\mathrm{C}(6)-\mathrm{H}(6 \mathrm{~B})$ & $0.96(2)$ \\
\hline $\mathrm{C}(6)-\mathrm{H}(6 \mathrm{C})$ & $0.97(2)$ \\
\hline$C(11)-H(11 A)$ & $0.90(2)$ \\
\hline$C(11)-H(11 B)$ & $0.91(2)$ \\
\hline$C(11)-H(11 C)$ & $0.90(2)$ \\
\hline $\mathrm{C}(12)-\mathrm{H}(12 \mathrm{~A})$ & $0.95(3)$ \\
\hline $\mathrm{C}(12)-\mathrm{H}(12 \mathrm{~B})$ & $0.95(3)$ \\
\hline $\mathrm{C}(12)-\mathrm{H}(12 \mathrm{C})$ & $0.95(3)$ \\
\hline
\end{tabular}


(C) 2006 American Chemical Society, Organometallics, Bleeke om058058f Supporting Info Page 82

\begin{tabular}{|c|c|}
\hline$C(13)-H(13 A)$ & $0.90(3)$ \\
\hline$C(13)-H(13 B)$ & $0.90(3)$ \\
\hline $\mathrm{C}(13)-\mathrm{H}(13 \mathrm{C})$ & $0.90(3)$ \\
\hline$C(21)-H(21 A)$ & $0.95(3)$ \\
\hline $\mathrm{C}(21)-\mathrm{H}(21 \mathrm{~B})$ & $0.94(3)$ \\
\hline $\mathrm{C}(21)-\mathrm{H}(21 \mathrm{C})$ & $0.95(3)$ \\
\hline $\mathrm{C}(22)-\mathrm{H}(22 \mathrm{~A})$ & $0.88(3)$ \\
\hline $\mathrm{C}(22)-\mathrm{H}(22 \mathrm{~B})$ & $0.88(2)$ \\
\hline $\mathrm{C}(22)-\mathrm{H}(22 \mathrm{C})$ & $0.89(2)$ \\
\hline $\mathrm{C}(23)-\mathrm{H}(23 \mathrm{~A})$ & $0.96(3)$ \\
\hline $\mathrm{C}(23)-\mathrm{H}(23 \mathrm{~B})$ & $0.95(3)$ \\
\hline $\mathrm{C}(23)-\mathrm{H}(23 \mathrm{C})$ & $0.95(3)$ \\
\hline$C(4)-\operatorname{Rh}(1)-C(1)$ & $80.70(15)$ \\
\hline$C(4)-R h(1)-P(1)$ & $98.31(10)$ \\
\hline$C(1)-R h(1)-P(1)$ & $90.75(12)$ \\
\hline$C(4)-R h(1)-P(2)$ & $154.50(10)$ \\
\hline$C(1)-R h(1)-P(2)$ & $87.48(14)$ \\
\hline $\mathrm{P}(1)-\operatorname{Rh}(1)-\mathrm{P}(2)$ & $104.40(3)$ \\
\hline$C(4)-R h(1)-S(1)$ & $46.31(10)$ \\
\hline$C(1)-R h(1)-S(1)$ & $91.40(9)$ \\
\hline $\mathrm{P}(1)-\operatorname{Rh}(1)-\mathrm{S}(1)$ & $143.47(4)$ \\
\hline$P(2)-\operatorname{Rh}(1)-S(1)$ & $112.12(4)$ \\
\hline$C(4)-R h(1)-S(1) \# 1$ & $96.61(9)$ \\
\hline $\mathrm{C}(1)-\mathrm{Rh}(1)-\mathrm{S}(1) \# 1$ & $174.76(10)$ \\
\hline $\mathrm{P}(1)-\mathrm{Rh}(1)-\mathrm{S}(1) \# 1$ & $94.11(3)$ \\
\hline$P(2)-\operatorname{Rh}(1)-S(1) \# 1$ & $93.22(4)$ \\
\hline$S(1)-\operatorname{Rh}(1)-S(1) \# 1$ & $83.52(3)$ \\
\hline $\mathrm{C}(4)-\mathrm{S}(1)-\mathrm{Rh}(1)$ & $57.78(10)$ \\
\hline$C(4)-S(1)-R h(1) \# 1$ & $108.36(11)$ \\
\hline$R h(1)-S(1)-R h(1) \# 1$ & $96.44(3)$ \\
\hline$C(11)-P(1)-C(12)$ & $101.4(2)$ \\
\hline$C(11)-P(1)-C(13)$ & $100.5(2)$ \\
\hline$C(12) \cdot P(1)-C(13)$ & $99.6(2)$ \\
\hline$C(11)-P(1)-\operatorname{Rh}(1)$ & $117.45(15)$ \\
\hline $\mathrm{C}(12)-\mathrm{P}(1)-\mathrm{Rh}(1)$ & $120.27(15)$ \\
\hline
\end{tabular}


(C) 2006 American Chemical Society, Organometallics, Bleeke om058058f Supporting Info Page 83

\begin{tabular}{|c|c|}
\hline$C(13)-P(1)-\operatorname{Rh}(1)$ & $114.36(14)$ \\
\hline$C(22)-P(2)-C(21)$ & $101.2(2)$ \\
\hline$C(22)-P(2)-C(23)$ & $102.7(2)$ \\
\hline$C(21)-P(2)-C(23)$ & $98.9(2)$ \\
\hline$C(22)-P(2)-\operatorname{Rh}(1)$ & $119.82(15)$ \\
\hline$C(21)-P(2)-R h(1)$ & $117.70(18)$ \\
\hline$C(23)-P(2)-R h(1)$ & $113.45(15)$ \\
\hline$C(2)-C(1)-R h(1)$ & $111.0(3)$ \\
\hline$C(2)-C(1)-H(1 A)$ & $114(2)$ \\
\hline $\mathrm{Rh}(1)-\mathrm{C}(1)-\mathrm{H}(1 \mathrm{~A})$ & $115(2)$ \\
\hline$C(2)-C(1)-H(1 B)$ & $108(3)$ \\
\hline $\operatorname{Rh}(1)-C(1)-H(1 B)$ & $107(3)$ \\
\hline$H(1 A)-C(1)-H(1 B)$ & $100(4)$ \\
\hline$C(3)-C(2)-C(1)$ & $117.8(3)$ \\
\hline$C(3)-C(2)-C(5)$ & $125.8(4)$ \\
\hline$C(1)-C(2)-C(5)$ & $116.4(3)$ \\
\hline$C(2)-C(3)-C(4)$ & $117.5(3)$ \\
\hline$C(2)-C(3)-C(6)$ & $125.5(3)$ \\
\hline$C(4)-C(3)-C(6)$ & $116.9(3)$ \\
\hline$C(3)-C(4)-S(1)$ & $114.8(2)$ \\
\hline$C(3)-C(4)-R h(1)$ & $111.7(2)$ \\
\hline$S(1)-C(4)-R h(1)$ & $75.91(13)$ \\
\hline$C(3)-C(4)-H(4)$ & $112(2)$ \\
\hline $\mathrm{S}(1)-\mathrm{C}(4)-\mathrm{H}(4)$ & $118(2)$ \\
\hline $\operatorname{Rh}(1)-C(4)-H(4)$ & $120.4(19)$ \\
\hline$C(2)-C(5)-H(5 A)$ & $113(3)$ \\
\hline$C(2)-C(5)-H(5 B)$ & $114(3)$ \\
\hline$H(5 A)-C(5)-H(5 B)$ & $100(4)$ \\
\hline$C(2)-C(5)-H(5 C)$ & $112(3)$ \\
\hline $\mathrm{H}(5 \mathrm{~A})-\mathrm{C}(5)-\mathrm{H}(5 \mathrm{C})$ & $116(5)$ \\
\hline$H(5 B)-C(5)-H(5 C)$ & $101(5)$ \\
\hline$C(3)-C(6)-H(6 A)$ & $114(3)$ \\
\hline$C(3)-C(6)-H(6 B)$ & $110(3)$ \\
\hline$H(6 A)-C(6)-H(6 B)$ & $118(4)$ \\
\hline$C(3)-C(6)-H(6 C)$ & $112(3)$ \\
\hline$H(6 A)-C(6)-H(6 C)$ & $106(4)$ \\
\hline
\end{tabular}


(C) 2006 American Chemical Society, Organometallics, Bleeke om058058f Supporting Info Page 84

\begin{tabular}{|c|c|}
\hline$H(6 B)-C(6)-H(6 C)$ & $96(4)$ \\
\hline$P(1)-C(11)-H(11 A)$ & $107(3)$ \\
\hline$P(1)-C(11)-H(11 B)$ & $111(3)$ \\
\hline $\mathrm{H}(11 \mathrm{~A})-\mathrm{C}(11)-\mathrm{H}(11 \mathrm{~B})$ & $117(4)$ \\
\hline$P(1)-C(11)-H(11 C)$ & $110(3)$ \\
\hline $\mathrm{H}(11 \mathrm{~A})-\mathrm{C}(11)-\mathrm{H}(11 \mathrm{C})$ & $109(4)$ \\
\hline $\mathrm{H}(11 \mathrm{~B})-\mathrm{C}(11)-\mathrm{H}(11 \mathrm{C})$ & $102(4)$ \\
\hline $\mathrm{P}(1)-\mathrm{C}(12)-\mathrm{H}(12 \mathrm{~A})$ & $112(3)$ \\
\hline$P(1)-C(12)-H(12 B)$ & $110(3)$ \\
\hline $\mathrm{H}(12 \mathrm{~A})-\mathrm{C}(12)-\mathrm{H}(12 \mathrm{~B})$ & $111(4)$ \\
\hline$P(1)-C(12)-H(12 C)$ & $111(3)$ \\
\hline $\mathrm{H}(12 \mathrm{~A})-\mathrm{C}(12)-\mathrm{H}(12 \mathrm{C})$ & $111(4)$ \\
\hline $\mathrm{H}(12 \mathrm{~B})-\mathrm{C}(12)-\mathrm{H}(12 \mathrm{C})$ & $101(5)$ \\
\hline$P(1)-C(13)-H(13 A)$ & $113(3)$ \\
\hline$P(1)-C(13)-H(13 B)$ & $108(3)$ \\
\hline $\mathrm{H}(13 \mathrm{~A})-\mathrm{C}(13)-\mathrm{H}(13 \mathrm{~B})$ & $110(4)$ \\
\hline$P(1)-C(13) \cdot H(13 C)$ & $104(3)$ \\
\hline $\mathrm{H}(13 \mathrm{~A})-\mathrm{C}(13)-\mathrm{H}(13 \mathrm{C})$ & $109(4)$ \\
\hline$H(13 B)-C(13)-H(13 C)$ & $114(4)$ \\
\hline$P(2)-C(21)-H(21 A)$ & $111(3)$ \\
\hline $\mathrm{P}(2)-\mathrm{C}(21)-\mathrm{H}(21 \mathrm{~B})$ & $117(3)$ \\
\hline$H(21 A)-C(21)-H(21 B)$ & $104(4)$ \\
\hline $\mathrm{P}(2)-\mathrm{C}(21)-\mathrm{H}(21 \mathrm{C})$ & $105(3)$ \\
\hline $\mathrm{H}(21 \mathrm{~A})-\mathrm{C}(21)-\mathrm{H}(21 \mathrm{C})$ & $108(5)$ \\
\hline $\mathrm{H}(21 \mathrm{~B})-\mathrm{C}(21)-\mathrm{H}(21 \mathrm{C})$ & $111(4)$ \\
\hline $\mathrm{P}(2)-\mathrm{C}(22)-\mathrm{H}(22 \mathrm{~A})$ & $110(3)$ \\
\hline$P(2)-C(22)-H(22 B)$ & $109(3)$ \\
\hline $\mathrm{H}(22 \mathrm{~A})-\mathrm{C}(22)-\mathrm{H}(22 \mathrm{~B})$ & $114(5)$ \\
\hline $\mathrm{P}(2)-\mathrm{C}(22)-\mathrm{H}(22 \mathrm{C})$ & $111(3)$ \\
\hline $\mathrm{H}(22 \mathrm{~A})-\mathrm{C}(22)-\mathrm{H}(22 \mathrm{C})$ & $107(5)$ \\
\hline $\mathrm{H}(22 \mathrm{~B})-\mathrm{C}(22)-\mathrm{H}(22 \mathrm{C})$ & $107(4)$ \\
\hline$P(2)-C(23)-H(23 A)$ & $115(3)$ \\
\hline $\mathrm{P}(2)-\mathrm{C}(23)-\mathrm{H}(23 \mathrm{~B})$ & $113(3)$ \\
\hline $\mathrm{H}(23 \mathrm{~A})-\mathrm{C}(23)-\mathrm{H}(23 \mathrm{~B})$ & $110(4)$ \\
\hline$P(2)-C(23)-H(23 C)$ & $114(3)$ \\
\hline $\mathrm{H}(23 \mathrm{~A})-\mathrm{C}(23)-\mathrm{H}(23 \mathrm{C})$ & $102(4)$ \\
\hline
\end{tabular}


(C) 2006 American Chemical Society, Organometallics, Bleeke om058058f Supporting Info Page 85

$\mathrm{H}(23 \mathrm{~B})-\mathrm{C}(23)-\mathrm{H}(23 \mathrm{C})$

Symmetry transformations used to generate equivalent atoms:

$\# 1-x-1 / 2,-y-3 / 2, z$ 
(C) 2006 American Chemical Society, Organometallics, Bleeke om058058f Supporting Info Page 86

Table 4. Anisotropic displacement parameters $\left(\AA^{2} \times 10^{3}\right)$ for jb14704. The anisotropic displacement factor exponent takes the form: $-2 \pi^{2}\left[h^{2} a^{* 2} U^{11}+\ldots+2 h k a^{*} b^{*} U^{12}\right]$

\begin{tabular}{lcccccc}
\hline & $\mathrm{U}^{11}$ & $\mathrm{U}^{22}$ & $\mathrm{U}^{33}$ & $\mathrm{U}^{23}$ & $\mathrm{U}^{13}$ & $\mathrm{U}^{12}$ \\
\hline $\mathrm{Rh}(1)$ & $22(1)$ & $21(1)$ & $28(1)$ & $4(1)$ & $0(1)$ & $0(1)$ \\
$\mathrm{S}(1)$ & $23(1)$ & $22(1)$ & $29(1)$ & $0(1)$ & $1(1)$ & $-1(1)$ \\
$\mathrm{P}(1)$ & $31(1)$ & $24(1)$ & $37(1)$ & $2(1)$ & $5(1)$ & $3(1)$ \\
$\mathrm{P}(2)$ & $32(1)$ & $34(1)$ & $32(1)$ & $10(1)$ & $-5(1)$ & $-7(1)$ \\
$\mathrm{C}(1)$ & $27(2)$ & $30(2)$ & $41(2)$ & $11(2)$ & $2(2)$ & $-5(1)$ \\
$\mathrm{C}(2)$ & $27(2)$ & $27(2)$ & $47(2)$ & $1(2)$ & $3(2)$ & $3(1)$ \\
$\mathrm{C}(3)$ & $33(2)$ & $25(2)$ & $41(2)$ & $-1(2)$ & $10(2)$ & $-3(2)$ \\
$\mathrm{C}(4)$ & $26(2)$ & $23(2)$ & $29(2)$ & $5(1)$ & $2(1)$ & $-1(1)$ \\
$\mathrm{C}(5)$ & $28(2)$ & $50(3)$ & $83(4)$ & $10(3)$ & $11(2)$ & $-3(2)$ \\
$\mathrm{C}(6)$ & $43(2)$ & $40(2)$ & $48(2)$ & $11(2)$ & $13(2)$ & $-3(2)$ \\
$\mathrm{C}(11)$ & $48(2)$ & $36(2)$ & $59(3)$ & $-2(3)$ & $21(3)$ & $5(2)$ \\
$\mathrm{C}(12)$ & $63(3)$ & $26(2)$ & $54(3)$ & $2(2)$ & $14(2)$ & $8(2)$ \\
$\mathrm{C}(13)$ & $54(3)$ & $47(3)$ & $47(3)$ & $-18(2)$ & $-3(2)$ & $13(2)$ \\
$\mathrm{C}(21)$ & $51(3)$ & $40(2)$ & $57(3)$ & $21(2)$ & $-20(2)$ & $-2(2)$ \\
$\mathrm{C}(22)$ & $50(3)$ & $58(3)$ & $38(2)$ & $15(2)$ & $-2(2)$ & $-21(2)$ \\
$\mathrm{C}(23)$ & $50(2)$ & $55(3)$ & $38(2)$ & $3(2)$ & $-7(2)$ & $-9(2)$ \\
& & & & & & \\
\hline
\end{tabular}


(C) 2006 American Chemical Society, Organometallics, Bleeke om058058f Supporting Info Page 87

Table 5. Hydrogen coordinates $\left(\times 10^{4}\right)$ and isotropic displacement parameters $\left(\AA^{2} \times 10^{3}\right)$ for jb14704.

\begin{tabular}{|c|c|c|c|c|}
\hline & $x$ & $\mathrm{y}$ & $\mathrm{z}$ & $U(\mathrm{eq})$ \\
\hline $\mathrm{H}(\mathrm{IA})$ & $-4287(13)$ & $-6995(9)$ & $5000(40)$ & 39 \\
\hline $\mathrm{H}(1 \mathrm{~B})$ & $-4148(18)$ & $-7369(12)$ & $4300(30)$ & 39 \\
\hline $\mathrm{H}(4)$ & $-2968(14)$ & $-7606(10)$ & $7600(50)$ & 31 \\
\hline$H(5 A)$ & $-5040(20)$ & $-7786(13)$ & $7110(40)$ & 80 \\
\hline $\mathrm{H}(5 \mathrm{~B})$ & $-5100(20)$ & $-7729(14)$ & $5620(40)$ & 80 \\
\hline $\mathrm{H}(5 \mathrm{C})$ & $-5150(20)$ & $-7334(11)$ & $6390(60)$ & 80 \\
\hline $\mathrm{H}(6 \mathrm{~A})$ & $-4396(11)$ & $-8037(15)$ & $8530(50)$ & 65 \\
\hline $\mathrm{H}(6 \mathrm{~B})$ & $-3737(19)$ & $-7903(14)$ & $9140(40)$ & 65 \\
\hline $\mathrm{H}(6 \mathrm{C})$ & $-3801(18)$ & $-8265(9)$ & $8220(50)$ & 65 \\
\hline$H(11 A)$ & $-3910(20)$ & $-6285(10)$ & $8160(50)$ & 72 \\
\hline$H(11 B)$ & $-3960(20)$ & $-6780(10)$ & $8230(40)$ & 72 \\
\hline $\mathrm{H}(11 \mathrm{C})$ & $-4252(15)$ & $-6548(16)$ & $7150(40)$ & 72 \\
\hline $\mathrm{H}(12 \mathrm{~A})$ & $-3290(20)$ & $-5802(12)$ & $6500(40)$ & 71 \\
\hline $\mathrm{H}(12 \mathrm{~B})$ & $-3587(16)$ & $-5994(14)$ & $5150(50)$ & 71 \\
\hline $\mathrm{H}(12 \mathrm{C})$ & $-2936(15)$ & $-5980(13)$ & $5210(50)$ & 71 \\
\hline $\mathrm{H}(13 \mathrm{~A})$ & $-2740(20)$ & $-6248(11)$ & $8460(50)$ & 74 \\
\hline $\mathrm{H}(13 \mathrm{~B})$ & $-2327(15)$ & $-6456(15)$ & $7450(50)$ & 74 \\
\hline $\mathrm{H}(13 \mathrm{C})$ & $-2700(20)$ & $-6719(11)$ & $8450(50)$ & 74 \\
\hline $\mathrm{H}(21 \mathrm{~A})$ & $-3850(20)$ & $-6487(15)$ & $1480(30)$ & 74 \\
\hline $\mathrm{H}(21 \mathrm{~B})$ & $-3820(20)$ & $-6248(9)$ & $2860(50)$ & 74 \\
\hline $\mathrm{H}(21 \mathrm{C})$ & $-4168(14)$ & $-6682(14)$ & $2780(60)$ & 74 \\
\hline $\mathrm{H}(22 \mathrm{~A})$ & $-2640(20)$ & $-6526(15)$ & $1270(30)$ & 73 \\
\hline $\mathrm{H}(22 \mathrm{~B})$ & $-2241(15)$ & $-6713(14)$ & $2360(50)$ & 73 \\
\hline $\mathrm{H}(22 \mathrm{C})$ & $-2540(20)$ & $-6319(10)$ & $2590(50)$ & 73 \\
\hline $\mathrm{H}(23 \mathrm{~A})$ & $-3310(20)$ & $-7194(14)$ & $620(30)$ & 72 \\
\hline$H(23 B)$ & $-3664(15)$ & $-7460(14)$ & $1760(50)$ & 72 \\
\hline $\mathrm{H}(23 \mathrm{C})$ & $-3014(17)$ & $-7506(13)$ & $1570(60)$ & 72 \\
\hline
\end{tabular}


(C) 2006 American Chemical Society, Organometallics, Bleeke om058058f Supporting Info Page 88

\section{compound 12a}

Table 1. Crystal data and structure refinement for jb13104.

Identification code

Empirical formula

Formula weight

Temperature

Wavelength

Crystal system

Space group

Unit cell dimensions

Volume

Z

Density (calculated)

Absorption coefficient

$F(000)$

Crystal size

Theta range for data collection

Index ranges

Reflections collected

Independent reflections

Completeness to theta $=29.00^{\circ}$

Absorption correction

Max. and min. transmission

Refinement method

Data / restraints / parameters

Goodness-of-fit on $\mathrm{F}^{2}$

Final $\mathrm{R}$ indices [I $>2 \operatorname{sigma}(\mathrm{I})]$

$\mathrm{R}$ indices (all data)

Largest diff. peak and hole j13104/lt

$\mathrm{C}_{16} \mathrm{H}_{35} \mathrm{Cl}_{2} \mathrm{P}_{2} \mathrm{Rh} \mathrm{S}$

495.25

165(2) K

$0.71073 \AA$

Monoclinic

$\mathrm{P} 2 \mathrm{I} / \mathrm{n}$

$\mathrm{a}=10.41510(10) \AA$

$\alpha=90^{\circ}$.

$\mathrm{b}=19.0763(3) \AA$

$\beta=96.8390(10)^{\circ}$.

$\mathrm{c}=11.4420(2) \AA$

$\gamma=90^{\circ}$.
2257.14(6) $\AA^{3}$

4

$1.457 \mathrm{Mg} / \mathrm{m}^{3}$

$1.223 \mathrm{~mm}^{-1}$

1024

$0.28 \times 0.24 \times 0.24 \mathrm{~mm}^{3}$

2.09 to $29.00^{\circ}$.

$-14 \leq \mathrm{h} \leq 14,-26 \leq \mathrm{k} \leq 25,-15 \leq 1 \leq 15$

46030

$5996[\mathrm{R}(\mathrm{int})=0.0299]$

$99.9 \%$

Semi-empirical from equivalents

0.7578 and 0.7257

Full-matrix least-squares on $\mathrm{F}^{2}$

$5996 / 0 / 209$

1.059

$\mathrm{R} 1=0.0272, \mathrm{wR} 2=0.0561$

$\mathrm{R} 1=0.0342, \mathrm{wR} 2=0.0583$

0.572 and -0.654 e. $\AA^{-3}$ 
(C) 2006 American Chemical Society, Organometallics, Bleeke om058058f Supporting Info Page 89

Table 2. Atomic coordinates ( $\left.\times 10^{4}\right)$ and equivalent isotropic displacement parameters $\left(\AA^{2} \times 10^{3}\right)$ for jb13104. $U(e q)$ is defined as one third of the trace of the orthogonalized $U^{i j}$ tensor.

\begin{tabular}{|c|c|c|c|c|}
\hline & $\mathrm{x}$ & $y$ & $\mathrm{z}$ & $\mathrm{U}(\mathrm{eq})$ \\
\hline $\operatorname{Rh}(1)$ & $778(1)$ & $479(1)$ & $1294(1)$ & $17(1)$ \\
\hline$S(1)$ & $-1036(1)$ & $-290(1)$ & $786(1)$ & $20(1)$ \\
\hline$P(1)$ & $2678(1)$ & $-79(1)$ & $2051(1)$ & $24(1)$ \\
\hline$P(2)$ & $1304(1)$ & $1634(1)$ & $1249(1)$ & $22(1)$ \\
\hline$C(1)$ & $310(2)$ & $554(1)$ & $3024(2)$ & $22(1)$ \\
\hline$C(2)$ & $-1063(2)$ & $793(1)$ & $3025(2)$ & $24(1)$ \\
\hline$C(3)$ & $-1786(2)$ & $870(1)$ & $1990(2)$ & $23(1)$ \\
\hline$C(4)$ & $-1200(2)$ & $639(1)$ & $925(2)$ & $20(1)$ \\
\hline$C(5)$ & $-1516(2)$ & $920(1)$ & $4213(2)$ & $36(1)$ \\
\hline$C(6)$ & $-3155(2)$ & $1141(1)$ & $1812(2)$ & $38(1)$ \\
\hline$C(11)$ & $3577(2)$ & $288(1)$ & $3382(2)$ & $41(1)$ \\
\hline$C(12)$ & $3992(2)$ & $-214(2)$ & $1153(2)$ & $41(1)$ \\
\hline$C(13)$ & $2419(3)$ & $-972(1)$ & $2554(2)$ & $41(1)$ \\
\hline$C(21)$ & $316(2)$ & $2062(1)$ & $11(2)$ & $28(1)$ \\
\hline$C(22)$ & $664(2)$ & $2813(1)$ & $-304(2)$ & $38(1)$ \\
\hline$C(23)$ & $951(2)$ & $2193(1)$ & $2492(2)$ & $32(1)$ \\
\hline$C(24)$ & $2007(3)$ & $2221(1)$ & $3535(2)$ & $43(1)$ \\
\hline$C(25)$ & $2959(2)$ & $1905(1)$ & $1040(2)$ & $33(1)$ \\
\hline$C(26)$ & $3400(2)$ & $1679(1)$ & $-125(2)$ & $42(1)$ \\
\hline$C(1 S)$ & $-1708(3)$ & $-1093(1)$ & $3499(2)$ & $45(1)$ \\
\hline $\mathrm{Cl}(1)$ & $-900(5)$ & $-1869(3)$ & $3297(5)$ & $57(1)$ \\
\hline $\mathrm{Cl}(2)$ & $-3373(1)$ & $-1177(1)$ & $3538(1)$ & $88(1)$ \\
\hline $\mathrm{Cl}\left(1^{\prime}\right)$ & $-1180(60)$ & $-1965(19)$ & $3285(16)$ & $87(5)$ \\
\hline
\end{tabular}


(C) 2006 American Chemical Society, Organometallics, Bleeke om058058f Supporting Info Page 90

Table 3. Bond lengths $[\AA]$ and angles $\left[{ }^{\circ}\right]$ for jb13104.

\begin{tabular}{|c|c|}
\hline $\operatorname{Rh}(1)-C(4)$ & $2.0758(18)$ \\
\hline $\mathrm{Rh}(1)-\mathrm{C}(1)$ & $2.0987(18)$ \\
\hline $\mathrm{Rh}(1)-\mathrm{P}(2)$ & $2.2708(5)$ \\
\hline $\operatorname{Rh}(1)-P(1)$ & $2.3229(5)$ \\
\hline$R h(1)-S(1)$ & $2.4075(5)$ \\
\hline $\operatorname{Rh}(1)-S(1) \# 1$ & $2.4534(5)$ \\
\hline$S(1)-C(4)$ & $1.7891(18)$ \\
\hline$S(1)-\operatorname{Rh}(1) \# 1$ & $2.4534(5)$ \\
\hline$P(1)-C(12)$ & $1.824(2)$ \\
\hline$P(1)-C(13)$ & $1.828(2)$ \\
\hline$P(1)-C(11)$ & $1.829(2)$ \\
\hline$P(2)-C(21)$ & $1.840(2)$ \\
\hline$P(2)-C(25)$ & $1.843(2)$ \\
\hline$P(2)-C(23)$ & $1.850(2)$ \\
\hline$C(1)-C(2)$ & $1.501(3)$ \\
\hline$C(2)-C(3)$ & $1.333(3)$ \\
\hline$C(2)-C(5)$ & $1.510(3)$ \\
\hline$C(3)-C(4)$ & $1.493(3)$ \\
\hline$C(3)-C(6)$ & $1.507(3)$ \\
\hline$C(21)-C(22)$ & $1.532(3)$ \\
\hline$C(23)-C(24)$ & $1.524(3)$ \\
\hline$C(25)-C(26)$ & $1.523(3)$ \\
\hline$C(1 S)-C l(1)$ & $1.731(7)$ \\
\hline$C(1 S)-C l(2)$ & $1.748(3)$ \\
\hline $\mathrm{C}(1 \mathrm{~S})-\mathrm{Cl}\left(1^{\prime}\right)$ & $1.78(2)$ \\
\hline$C(4)-R h(1)-C(1)$ & $81.12(7)$ \\
\hline$C(4)-R h(1)-P(2)$ & $95.13(5)$ \\
\hline$C(1)-R h(1)-P(2)$ & $92.25(5)$ \\
\hline$C(4)-R h(1)-P(1)$ & $157.55(5)$ \\
\hline$C(1)-\operatorname{Rh}(1)-P(1)$ & $87.86(5)$ \\
\hline$P(2)-R h(1)-P(1)$ & $104.847(19)$ \\
\hline$C(4)-R h(1)-S(1)$ & $46.31(5)$ \\
\hline$C(1)-\operatorname{Rh}(1)-S(1)$ & $90.31(5)$ \\
\hline
\end{tabular}


(C) 2006 American Chemical Society, Organometallics, Bleeke om058058f Supporting Info Page 91

\begin{tabular}{|c|c|}
\hline$P(2)-R h(1)-S(1)$ & $140.338(18)$ \\
\hline$P(1)-R h(1)-S(1)$ & $114.799(18)$ \\
\hline$C(4)-R h(1)-S(1) \# 1$ & $92.58(5)$ \\
\hline $\mathrm{C}(1)-\mathrm{Rh}(1)-\mathrm{S}(1) \# 1$ & $171.62(5)$ \\
\hline$P(2)-\operatorname{Rh}(1)-S(1) \# 1$ & $93.811(17)$ \\
\hline$P(1)-R h(1)-S(1) \# 1$ & $96.156(17)$ \\
\hline$S(1)-\operatorname{Rh}(1)-S(1) \# 1$ & $81.331(16)$ \\
\hline$C(4)-S(1)-R h(1)$ & $57.03(6)$ \\
\hline$C(4)-S(1)-R h(1) \# 1$ & $104.77(6)$ \\
\hline $\operatorname{Rh}(1)-S(1)-\operatorname{Rh}(1) \# 1$ & $98.669(16)$ \\
\hline$C(12) \cdot P(1)-C(13)$ & $101.28(12)$ \\
\hline$C(12)-P(1)-C(11)$ & $100.31(11)$ \\
\hline$C(13) \cdot P(1)-C(11)$ & $100.06(12)$ \\
\hline$C(12)-P(1)-R h(1)$ & $121.15(8)$ \\
\hline$C(13)-P(1)-R h(1)$ & $113.18(8)$ \\
\hline$C(11)-P(1)-R h(1)$ & $117.61(8)$ \\
\hline$C(21)-P(2)-C(25)$ & $102.79(10)$ \\
\hline$C(21)-P(2)-C(23)$ & $101.05(10)$ \\
\hline$C(25)-P(2)-C(23)$ & $102.25(10)$ \\
\hline$C(21)-P(2)-R h(1)$ & $109.50(7)$ \\
\hline$C(25)-P(2)-R h(1)$ & $120.48(8)$ \\
\hline$C(23)-P(2)-R h(1)$ & $118.13(7)$ \\
\hline$C(2)-C(1)-\operatorname{Rh}(1)$ & $110.47(12)$ \\
\hline$C(3)-C(2)-C(1)$ & $118.02(17)$ \\
\hline$C(3)-C(2)-C(5)$ & $125.28(19)$ \\
\hline$C(1)-C(2)-C(5)$ & $116.70(17)$ \\
\hline$C(2)-C(3)-C(4)$ & $116.75(17)$ \\
\hline$C(2)-C(3)-C(6)$ & $125.69(18)$ \\
\hline$C(4)-C(3)-C(6)$ & $117.51(17)$ \\
\hline$C(3)-C(4)-S(1)$ & $114.70(13)$ \\
\hline$C(3)-C(4)-\operatorname{Rh}(1)$ & $111.68(13)$ \\
\hline$S(1)-C(4)-R h(1)$ & $76.66(7)$ \\
\hline$C(22)-C(21)-P(2)$ & $118.06(15)$ \\
\hline$C(24)-C(23)-P(2)$ & $115.50(17)$ \\
\hline$C(26)-C(25)-P(2)$ & $114.73(15)$ \\
\hline $\mathrm{Cl}(1)-\mathrm{C}(1 \mathrm{~S})-\mathrm{Cl}(2)$ & $115.1(2)$ \\
\hline
\end{tabular}


$\mathrm{Cl}(1)-\mathrm{C}(1 \mathrm{~S})-\mathrm{Cl}\left(1^{\prime}\right)$

$\mathrm{Cl}(2)-\mathrm{C}(1 \mathrm{~S})-\mathrm{Cl}\left(1^{\prime}\right)$

Symmetry transformations used to generate equivalent atoms:

$\# 1-x,-y,-z$ 
(C) 2006 American Chemical Society, Organometallics, Bleeke om058058f Supporting Info Page 93

Table 4. Anisotropic displacement parameters $\left(\AA^{2} \times 10^{3}\right)$ for jb13104. The anisotropic displacement factor exponent takes the form: $-2 \pi^{2}\left[h^{2} a^{* 2} U^{11}+\ldots+2 h k a^{*} b^{*} U^{12}\right]$

\begin{tabular}{|c|c|c|c|c|c|c|}
\hline & $U^{\prime \prime}$ & $\mathrm{U}^{22}$ & $\mathrm{U}^{33}$ & $\mathrm{U}^{23}$ & $\mathrm{U}^{13}$ & $\mathrm{U}^{12}$ \\
\hline $\operatorname{Rh}(1)$ & $18(1)$ & $15(1)$ & $17(1)$ & $0(1)$ & $2(1)$ & $-1(1)$ \\
\hline$S(1)$ & $23(1)$ & $17(1)$ & $19(1)$ & $-1(1)$ & $4(1)$ & $-3(1)$ \\
\hline$P(1)$ & $23(1)$ & $25(1)$ & $22(1)$ & $-2(1)$ & $0(1)$ & $4(1)$ \\
\hline $\mathrm{P}(2)$ & $25(1)$ & $18(1)$ & $22(1)$ & $-1(1)$ & $1(1)$ & $-4(1)$ \\
\hline$C(1)$ & $27(1)$ & $22(1)$ & $18(1)$ & $1(1)$ & $2(1)$ & $1(1)$ \\
\hline$C(2)$ & $29(1)$ & $20(1)$ & $24(1)$ & $-3(1)$ & $7(1)$ & $1(1)$ \\
\hline$C(3)$ & $23(1)$ & $19(1)$ & $27(1)$ & $-4(1)$ & $6(1)$ & $0(1)$ \\
\hline$C(4)$ & $22(1)$ & $18(1)$ & $21(1)$ & $-1(1)$ & $2(1)$ & $-1(1)$ \\
\hline$C(5)$ & $38(1)$ & $43(1)$ & $27(1)$ & $-4(1)$ & $9(1)$ & $5(1)$ \\
\hline$C(6)$ & $28(1)$ & $50(1)$ & $35(1)$ & $-14(1)$ & $4(1)$ & $9(1)$ \\
\hline$C(11)$ & $37(1)$ & $46(1)$ & $35(1)$ & $-9(1)$ & $-11(1)$ & $10(1)$ \\
\hline$C(12)$ & $23(1)$ & $66(2)$ & $35(1)$ & $-6(1)$ & $3(1)$ & $5(1)$ \\
\hline$C(13)$ & $50(1)$ & $27(1)$ & $43(1)$ & $7(1)$ & $2(1)$ & $12(1)$ \\
\hline$C(21)$ & $32(1)$ & $22(1)$ & $29(1)$ & $5(1)$ & $-1(1)$ & $-3(1)$ \\
\hline$C(22)$ & $47(1)$ & $25(1)$ & $41(1)$ & $10(1)$ & $1(1)$ & $-4(1)$ \\
\hline$C(23)$ & $43(1)$ & $22(1)$ & $31(1)$ & $-4(1)$ & $3(1)$ & $-3(1)$ \\
\hline$C(24)$ & $59(2)$ & $35(1)$ & $32(1)$ & $-5(1)$ & $-5(1)$ & $-8(1)$ \\
\hline$C(25)$ & $27(1)$ & $32(1)$ & $38(1)$ & $2(1)$ & $-1(1)$ & $-9(1)$ \\
\hline$C(26)$ & $32(1)$ & $48(1)$ & $49(1)$ & $0(1)$ & $12(1)$ & $-10(1)$ \\
\hline$C(1 S)$ & $52(2)$ & $47(2)$ & $37(1)$ & $7(1)$ & $8(1)$ & $-6(1)$ \\
\hline $\mathrm{Cl}(1)$ & $65(3)$ & $57(2)$ & $49(1)$ & $-1(1)$ & $7(1)$ & $5(1)$ \\
\hline $\mathrm{Cl}(2)$ & $48(1)$ & $149(1)$ & $67(1)$ & $3(1)$ & $6(1)$ & $5(1)$ \\
\hline $\mathrm{Cl}\left(1^{\prime}\right)$ & $151(12)$ & $50(6)$ & $63(4)$ & $7(3)$ & $19(7)$ & $34(8)$ \\
\hline
\end{tabular}


(C) 2006 American Chemical Society, Organometallics, Bleeke om058058f Supporting Info Page 94

Table 5. Hydrogen coordinates ( $\left.\times 10^{4}\right)$ and isotropic displacement parameters $\left(\AA^{2} \times 10^{3}\right)$ for jb13104.

\begin{tabular}{|c|c|c|c|c|}
\hline & $x$ & $y$ & $z$ & $\mathrm{U}(\mathrm{eq})$ \\
\hline $\mathrm{H}(1 \mathrm{~A})$ & 425 & 91 & 3413 & 26 \\
\hline $\mathrm{H}(1 \mathrm{~B})$ & 901 & 891 & 3474 & 26 \\
\hline $\mathrm{H}(4 \mathrm{~A})$ & -1490 & 899 & 181 & 24 \\
\hline $\mathrm{H}(5 \mathrm{~A})$ & -2421 & 1072 & 4105 & 53 \\
\hline $\mathrm{H}(5 \mathrm{~B})$ & -981 & 1284 & 4632 & 53 \\
\hline $\mathrm{H}(5 \mathrm{C})$ & -1439 & 485 & 4672 & 53 \\
\hline $\mathrm{H}(6 \mathrm{~A})$ & -3421 & 1276 & 2573 & 56 \\
\hline $\mathrm{H}(6 \mathrm{~B})$ & -3731 & 772 & 1457 & 56 \\
\hline $\mathrm{H}(6 \mathrm{C})$ & -3202 & 1549 & 1289 & 56 \\
\hline $\mathrm{H}(11 \mathrm{~A})$ & 4354 & 6 & 3607 & 61 \\
\hline $\mathrm{H}(11 \mathrm{~B})$ & 3030 & 283 & 4022 & 61 \\
\hline $\mathrm{H}(11 \mathrm{C})$ & 3829 & 771 & 3229 & 61 \\
\hline $\mathrm{H}(12 \mathrm{~A})$ & 4708 & -457 & 1618 & 62 \\
\hline $\mathrm{H}(12 \mathrm{~B})$ & 4293 & 241 & 895 & 62 \\
\hline $\mathrm{H}(12 \mathrm{C})$ & 3681 & -497 & 462 & 62 \\
\hline $\mathrm{H}(13 \mathrm{~A})$ & 3251 & -1180 & 2862 & 61 \\
\hline $\mathrm{H}(13 \mathrm{~B})$ & 2018 & -1254 & 1893 & 61 \\
\hline $\mathrm{H}(13 \mathrm{C})$ & 1849 & -961 & 3176 & 61 \\
\hline $\mathrm{H}(21 \mathrm{~A})$ & 352 & 1767 & -697 & 34 \\
\hline $\mathrm{H}(21 \mathrm{~B})$ & -592 & 2062 & 185 & 34 \\
\hline $\mathrm{H}(22 \mathrm{~A})$ & 61 & 2977 & -971 & 57 \\
\hline $\mathrm{H}(22 \mathrm{~B})$ & 1548 & 2824 & -516 & 57 \\
\hline $\mathrm{H}(22 \mathrm{C})$ & 606 & 3120 & 375 & 57 \\
\hline $\mathrm{H}(23 \mathrm{~A})$ & 786 & 2676 & 2196 & 38 \\
\hline $\mathrm{H}(23 \mathrm{~B})$ & 145 & 2022 & 2776 & 38 \\
\hline $\mathrm{H}(24 \mathrm{~A})$ & 1733 & 2529 & 4144 & 64 \\
\hline $\mathrm{H}(24 \mathrm{~B})$ & 2805 & 2403 & 3274 & 64 \\
\hline $\mathrm{H}(24 \mathrm{C})$ & 2161 & 1748 & 3856 & 64 \\
\hline $\mathrm{H}(25 \mathrm{~A})$ & 3014 & 2422 & 1097 & 39 \\
\hline $\mathrm{H}(25 \mathrm{~B})$ & 3565 & 1708 & 1690 & 39 \\
\hline
\end{tabular}


(C) 2006 American Chemical Society, Organometallics, Bleeke om058058f Supporting Info Page 95

\begin{tabular}{lrrrr}
$\mathrm{H}(26 \mathrm{~A})$ & 4287 & 1842 & -164 & 64 \\
$\mathrm{H}(26 \mathrm{~B})$ & 2825 & 1883 & -777 & 64 \\
$\mathrm{H}(26 \mathrm{C})$ & 3373 & 1166 & -184 & 64 \\
$\mathrm{H}(1 \mathrm{SA})$ & -1563 & -767 & 2854 & 54 \\
$\mathrm{H}(1 \mathrm{SB})$ & -1323 & -877 & 4246 & 54 \\
\hline
\end{tabular}


Table 1. Crystal data and structure refinement for jb13905.

\begin{tabular}{|c|c|c|}
\hline Identification code & \multicolumn{2}{|l|}{ j13905/lt/apex } \\
\hline Empirical formula & \multicolumn{2}{|c|}{$\mathrm{C}_{34} \mathrm{H}_{66} \mathrm{~N}_{2} \mathrm{P}_{2} \mathrm{Rh}_{2} \mathrm{~S}_{2}$} \\
\hline Formula weight & \multicolumn{2}{|l|}{834.77} \\
\hline Temperature & \multicolumn{2}{|l|}{$100(2) \mathrm{K}$} \\
\hline Wavelength & \multicolumn{2}{|l|}{$0.71073 \AA$} \\
\hline Crystal system & \multicolumn{2}{|l|}{ Monoclinic } \\
\hline Space group & \multicolumn{2}{|l|}{$\mathrm{P} 2(1) / \mathrm{n}$} \\
\hline \multirow[t]{3}{*}{ Unit cell dimensions } & $a=12.2598(5) \AA$ & $\alpha=90^{\circ}$ \\
\hline & $\mathrm{b}=12.0053(4) \AA$ & $\beta=90.583(2)^{\circ}$. \\
\hline & $c=13.5413(5) \AA$ & $\gamma=90^{\circ}$ \\
\hline Volume & \multicolumn{2}{|l|}{$1992.94(13) \AA^{3}$} \\
\hline Z & \multicolumn{2}{|l|}{2} \\
\hline Density (calculated) & \multicolumn{2}{|l|}{$1.391 \mathrm{Mg} / \mathrm{m}^{3}$} \\
\hline Absorption coefficient & \multicolumn{2}{|l|}{$1.037 \mathrm{~mm}^{-1}$} \\
\hline$F(000)$ & \multicolumn{2}{|l|}{872} \\
\hline Crystal size & \multicolumn{2}{|c|}{$0.19 \times 0.17 \times 0.10 \mathrm{~mm}^{3}$} \\
\hline Theta range for data collection & \multicolumn{2}{|l|}{2.23 to $35.49^{\circ}$} \\
\hline Index ranges & \multicolumn{2}{|c|}{$-17 \leq \mathrm{h} \leq 20,-13 \leq \mathrm{k} \leq 19,-18 \leq 1 \leq 22$} \\
\hline Reflections collected & \multicolumn{2}{|l|}{79728} \\
\hline Independent reflections & \multicolumn{2}{|c|}{$9061[R($ int $)=0.033]$} \\
\hline Completeness to theta $=35.49^{\circ}$ & \multicolumn{2}{|l|}{$99.7 \%$} \\
\hline Absorption correction & \multicolumn{2}{|c|}{ Semi-empirical from equivalents } \\
\hline Max. and min. transmission & \multicolumn{2}{|l|}{0.9034 and 0.8273} \\
\hline Refinement method & \multicolumn{2}{|c|}{ Full-matrix least-squares on $\mathrm{F}^{2}$} \\
\hline Data / restraints / parameters & \multicolumn{2}{|l|}{$9061 / 0 / 199$} \\
\hline Goodness-of-fit on $\mathrm{F}^{2}$ & \multicolumn{2}{|l|}{1.033} \\
\hline Final $R$ indices $[I>2 \operatorname{sigma}(I)]$ & \multicolumn{2}{|c|}{$\mathrm{R} 1=0.0275, \mathrm{wR} 2=0.0559$} \\
\hline $\mathrm{R}$ indices (all data) & \multicolumn{2}{|c|}{$\mathrm{R} 1=0.0383, \mathrm{wR} 2=0.0594$} \\
\hline Largest diff. peak and hole & \multicolumn{2}{|c|}{0.580 and $-0.730 \mathrm{e} . \AA^{-3}$} \\
\hline
\end{tabular}


(ㄷ) 2006 American Chemical Society, Organometallics, Bleeke om058058f Supporting Info Page 97

Table 2. Atomic coordinates ( $\left.\times 10^{4}\right)$ and equivalent isotropic displacement parameters $\left(\AA^{2} \times 10^{3}\right)$ for jb13905. $U(e q)$ is defined as one third of the trace of the orthogonalized $U^{i j}$ tensor.

\begin{tabular}{|c|c|c|c|c|}
\hline & $x$ & $y$ & $\mathrm{z}$ & $\mathrm{U}(\mathrm{eq})$ \\
\hline $\operatorname{Rh}(1)$ & $1447(1)$ & $4849(1)$ & $252(1)$ & $10(1)$ \\
\hline$S(1)$ & $105(1)$ & $4036(1)$ & $-813(1)$ & $12(1)$ \\
\hline$P(1)$ & $2326(1)$ & $4859(1)$ & $1725(1)$ & $11(1)$ \\
\hline$N(1)$ & $2547(1)$ & $6907(1)$ & $-758(1)$ & $19(1)$ \\
\hline$C(1)$ & $2649(1)$ & $3784(1)$ & $-309(1)$ & $15(1)$ \\
\hline$C(2)$ & $2311(1)$ & $2585(1)$ & $-188(1)$ & $17(1)$ \\
\hline$C(3)$ & $1295(1)$ & $2380(1)$ & $116(1)$ & $15(1)$ \\
\hline$C(4)$ & $583(1)$ & $3363(1)$ & $295(1)$ & $12(1)$ \\
\hline$C(5)$ & $3137(1)$ & $1715(1)$ & $-456(1)$ & $26(1)$ \\
\hline$C(6)$ & $780(1)$ & $1253(1)$ & $227(1)$ & $20(1)$ \\
\hline$C(7)$ & $2148(1)$ & $6172(1)$ & $-348(1)$ & $15(1)$ \\
\hline$C(8)$ & $3076(1)$ & $7742(1)$ & $-1364(1)$ & $30(1)$ \\
\hline$C(9)$ & $2213(2)$ & $8573(1)$ & $-1712(1)$ & $38(1)$ \\
\hline$C(10)$ & $3573(2)$ & $7122(2)$ & $-2230(2)$ & $67(1)$ \\
\hline$C(11)$ & $3785(5)$ & $8446(5)$ & $-599(5)$ & $39(1)$ \\
\hline$C\left(11^{\prime}\right)$ & $4077(5)$ & $8139(6)$ & $-889(5)$ & $49(2)$ \\
\hline$C(12)$ & $3373(1)$ & $5944(1)$ & $1884(1)$ & $18(1)$ \\
\hline$C(13)$ & $4392(1)$ & $5776(1)$ & $1263(1)$ & $24(1)$ \\
\hline$C(14)$ & $1455(1)$ & $5154(1)$ & $2789(1)$ & $17(1)$ \\
\hline$C(15)$ & $570(1)$ & $4281(1)$ & $2943(1)$ & $23(1)$ \\
\hline$C(16)$ & $3031(1)$ & $3572(1)$ & 2092(1) & $16(1)$ \\
\hline$C(17)$ & $3554(1)$ & $3562(1)$ & $3122(1)$ & $22(1)$ \\
\hline
\end{tabular}


(C) 2006 American Chemical Society, Organometallics, Bleeke om058058f Supporting Info Page 98

Table 3. Bond lengths $[\AA]$ and angles $\left[^{\circ}\right]$ for jb13905.

\begin{tabular}{|c|c|}
\hline $\mathrm{Rh}(1)-\mathrm{C}(7)$ & $1.9831(13)$ \\
\hline $\operatorname{Rh}(1)-C(4)$ & $2.0758(12)$ \\
\hline $\operatorname{Rh}(1)-C(1)$ & $2.0990(12)$ \\
\hline $\mathrm{Rh}(1)-\mathrm{P}(1)$ & $2.2568(3)$ \\
\hline $\operatorname{Rh}(1)-S(1)$ & $2.3859(3)$ \\
\hline $\operatorname{Rh}(1)-S(1) \# 1$ & $2.4531(3)$ \\
\hline$S(1)-C(4)$ & $1.7968(12)$ \\
\hline$S(1)-R h(1) \# 1$ & $2.4530(3)$ \\
\hline$P(1)-C(14)$ & $1.8364(13)$ \\
\hline$P(1)-C(16)$ & $1.8364(12)$ \\
\hline$P(1)-C(12)$ & $1.8402(13)$ \\
\hline$N(1)-C(7)$ & $1.1547(16)$ \\
\hline$N(1)-C(8)$ & $1.4523(18)$ \\
\hline$C(1)-C(2)$ & $1.5071(18)$ \\
\hline$C(1)-H(1 A)$ & 0.9900 \\
\hline $\mathrm{C}(1)-\mathrm{H}(1 \mathrm{~B})$ & 0.9900 \\
\hline$C(2)-C(3)$ & $1.3388(19)$ \\
\hline$C(2)-C(5)$ & $1.5024(18)$ \\
\hline$C(3)-C(4)$ & $1.4901(17)$ \\
\hline$C(3)-C(6)$ & $1.5019(18)$ \\
\hline $\mathrm{C}(4)-\mathrm{H}(4)$ & 1.0000 \\
\hline$C(5)-H(5 A)$ & 0.9800 \\
\hline $\mathrm{C}(5)-\mathrm{H}(5 \mathrm{~B})$ & 0.9800 \\
\hline $\mathrm{C}(5)-\mathrm{H}(5 \mathrm{C})$ & 0.9800 \\
\hline$C(6)-H(6 A)$ & 0.9800 \\
\hline$C(6)-H(6 B)$ & 0.9800 \\
\hline$C(6)-H(6 C)$ & 0.9800 \\
\hline$C(8)-C\left(11^{\prime}\right)$ & $1.460(7)$ \\
\hline$C(8)-C(10)$ & $1.521(3)$ \\
\hline$C(8)-C(9)$ & $1.526(2)$ \\
\hline$C(8)-C(11)$ & $1.589(7)$ \\
\hline$C(9)-H(9 A)$ & 0.9800 \\
\hline $\mathrm{C}(9)-\mathrm{H}(9 \mathrm{~B})$ & 0.9800 \\
\hline $\mathrm{C}(9)-\mathrm{H}(9 \mathrm{C})$ & 0.9800 \\
\hline
\end{tabular}


(C) 2006 American Chemical Society, Organometallics, Bleeke om058058f Supporting Info Page 99

\begin{tabular}{|c|c|}
\hline$C(10)-H(10 A)$ & 0.9800 \\
\hline $\mathrm{C}(10)-\mathrm{H}(10 \mathrm{~B})$ & 0.9800 \\
\hline $\mathrm{C}(10)-\mathrm{H}(10 \mathrm{C})$ & 0.9800 \\
\hline $\mathrm{C}(11)-\mathrm{H}(11 \mathrm{~A})$ & 0.9800 \\
\hline $\mathrm{C}(11)-\mathrm{H}(11 \mathrm{~B})$ & 0.9800 \\
\hline $\mathrm{C}(11)-\mathrm{H}(11 \mathrm{C})$ & 0.9800 \\
\hline$C\left(11^{\prime}\right)-H(11 D)$ & 0.9800 \\
\hline$C\left(11^{\prime}\right)-H(11 E)$ & 0.9800 \\
\hline $\mathrm{C}\left(11^{\prime}\right)-\mathrm{H}(11 \mathrm{~F})$ & 0.9800 \\
\hline$C(12)-C(13)$ & $1.526(2)$ \\
\hline $\mathrm{C}(12)-\mathrm{H}(12 \mathrm{~A})$ & 0.9900 \\
\hline $\mathrm{C}(12)-\mathrm{H}(12 \mathrm{~B})$ & 0.9900 \\
\hline$C(13)-H(13 A)$ & 0.9800 \\
\hline $\mathrm{C}(13)-\mathrm{H}(13 \mathrm{~B})$ & 0.9800 \\
\hline$C(13)-H(13 C)$ & 0.9800 \\
\hline$C(14)-C(15)$ & $1.524(2)$ \\
\hline $\mathrm{C}(14)-\mathrm{H}(14 \mathrm{~A})$ & 0.9900 \\
\hline $\mathrm{C}(14)-\mathrm{H}(14 \mathrm{~B})$ & 0.9900 \\
\hline$C(15)-H(15 A)$ & 0.9800 \\
\hline $\mathrm{C}(15)-\mathrm{H}(15 \mathrm{~B})$ & 0.9800 \\
\hline $\mathrm{C}(15)-\mathrm{H}(15 \mathrm{C})$ & 0.9800 \\
\hline$C(16)-C(17)$ & $1.5293(18$ \\
\hline$C(16)-H(16 A)$ & 0.9900 \\
\hline$C(16)-H(16 B)$ & 0.9900 \\
\hline$C(17)-H(17 A)$ & 0.9800 \\
\hline$C(17)-H(17 B)$ & 0.9800 \\
\hline $\mathrm{C}(17)-\mathrm{H}(17 \mathrm{C})$ & 0.9800 \\
\hline$C(7)-R h(1)-C(4)$ & $157.33(5)$ \\
\hline$C(7)-R h(1)-C(1)$ & $91.78(5)$ \\
\hline$C(4)-R h(1)-C(1)$ & $81.23(5)$ \\
\hline$C(7)-R h(1)-P(1)$ & $98.81(4)$ \\
\hline$C(4)-R h(1)-P(1)$ & $102.65(3)$ \\
\hline$C(1)-R h(1)-P(1)$ & $89.59(4)$ \\
\hline$C(7)-R h(1)-S(1)$ & $112.25(4)$ \\
\hline $\mathrm{C}(4)-\mathrm{Rh}(1)-\mathrm{S}(1)$ & $46.86(3)$ \\
\hline
\end{tabular}


(C) 2006 American Chemical Society, Organometallics, Bleeke om058058f Supporting Info Page 100

\begin{tabular}{|c|c|}
\hline$C(1)-R h(1)-S(1)$ & $90.83(4)$ \\
\hline $\mathrm{P}(1)-\mathrm{Rh}(1)-\mathrm{S}(1)$ & $148.903(11)$ \\
\hline$C(7)-\operatorname{Rh}(1)-S(1) \# 1$ & $91.74(4)$ \\
\hline$C(4)-\operatorname{Rh}(1)-S(1) \# 1$ & $93.55(3)$ \\
\hline$C(1)-R h(1)-S(1) \# 1$ & $173.67(4)$ \\
\hline$P(1)-R h(1)-S(1) \# 1$ & $95.068(11)$ \\
\hline$S(1)-\operatorname{Rh}(1)-S(1) \# 1$ & $82.972(11)$ \\
\hline$C(4)-S(1)-R h(1)$ & $57.46(4)$ \\
\hline$C(4)-S(1)-\operatorname{Rh}(1) \# 1$ & $103.54(4)$ \\
\hline $\operatorname{Rh}(1)-S(1)-\operatorname{Rh}(1) \# 1$ & $97.030(11)$ \\
\hline$C(14)-P(1)-C(16)$ & $103.06(6)$ \\
\hline$C(14)-P(1)-C(12)$ & $100.49(6)$ \\
\hline$C(16)-P(1)-C(12)$ & $103.78(6)$ \\
\hline$C(14)-P(1)-R h(1)$ & $114.71(5)$ \\
\hline$C(16)-P(1)-R h(1)$ & $116.98(4)$ \\
\hline$C(12)-P(1)-R h(1)$ & $115.67(4)$ \\
\hline$C(7)-N(1)-C(8)$ & $173.41(15)$ \\
\hline$C(2)-C(1)-R h(1)$ & $110.33(9)$ \\
\hline$C(2)-C(1)-H(1 A)$ & 109.6 \\
\hline $\mathrm{Rh}(1)-\mathrm{C}(1)-\mathrm{H}(\mathrm{IA})$ & 109.6 \\
\hline$C(2)-C(1)-H(1 B)$ & 109.6 \\
\hline $\mathrm{Rh}(1)-\mathrm{C}(1)-\mathrm{H}(1 \mathrm{~B})$ & 109.6 \\
\hline$H(1 A)-C(1)-H(1 B)$ & 108.1 \\
\hline$C(3)-C(2)-C(5)$ & $125.30(13)$ \\
\hline$C(3)-C(2)-C(1)$ & $117.83(11)$ \\
\hline$C(5)-C(2)-C(1)$ & $116.83(12)$ \\
\hline$C(2)-C(3)-C(4)$ & $116.95(11)$ \\
\hline$C(2)-C(3)-C(6)$ & $126.19(12)$ \\
\hline$C(4)-C(3)-C(6)$ & $116.72(11)$ \\
\hline$C(3)-C(4)-S(1)$ & $113.98(9)$ \\
\hline$C(3)-C(4)-R h(1)$ & $112.07(8)$ \\
\hline $\mathrm{S}(1)-\mathrm{C}(4)-\mathrm{Rh}(1)$ & $75.68(4)$ \\
\hline $\mathrm{C}(3)-\mathrm{C}(4)-\mathrm{H}(4)$ & 116.2 \\
\hline $\mathrm{S}(1)-\mathrm{C}(4)-\mathrm{H}(4)$ & 116.2 \\
\hline $\mathrm{Rh}(1)-\mathrm{C}(4)-\mathrm{H}(4)$ & 116.2 \\
\hline$C(2)-C(5)-H(5 A)$ & 109.5 \\
\hline
\end{tabular}


(C) 2006 American Chemical Society, Organometallics, Bleeke om058058f Supporting Info Page 101

\begin{tabular}{|c|c|}
\hline$C(2)-C(5) \cdot H(5 B)$ & 109.5 \\
\hline $\mathrm{H}(5 \mathrm{~A})-\mathrm{C}(5)-\mathrm{H}(5 \mathrm{~B})$ & 109.5 \\
\hline$C(2)-C(5)-H(5 C)$ & 109.5 \\
\hline $\mathrm{H}(5 \mathrm{~A})-\mathrm{C}(5)-\mathrm{H}(5 \mathrm{C})$ & 109.5 \\
\hline$H(5 B)-C(5)-H(5 C)$ & 109.5 \\
\hline$C(3)-C(6)-H(6 A)$ & 109.5 \\
\hline$C(3)-C(6)-H(6 B)$ & 109.5 \\
\hline $\mathrm{H}(6 \mathrm{~A})-\mathrm{C}(6)-\mathrm{H}(6 \mathrm{~B})$ & 109.5 \\
\hline$C(3)-C(6)-H(6 C)$ & 109.5 \\
\hline$H(6 A)-C(6)-H(6 C)$ & 109.5 \\
\hline $\mathrm{H}(6 \mathrm{~B})-\mathrm{C}(6)-\mathrm{H}(6 \mathrm{C})$ & 109.5 \\
\hline$N(1)-C(7)-R h(1)$ & $175.38(11)$ \\
\hline$N(1)-C(8)-C\left(11^{\prime}\right)$ & $110.8(3)$ \\
\hline$N(1)-C(8)-C(10)$ & $106.45(15)$ \\
\hline$C\left(11^{\prime}\right)-C(8)-C(10)$ & $99.1(3)$ \\
\hline$N(1)-C(8)-C(9)$ & $108.28(13)$ \\
\hline$C\left(1 l^{\prime}\right)-C(8)-C(9)$ & $120.0(3)$ \\
\hline$C(10)-C(8)-C(9)$ & $111.34(15)$ \\
\hline$N(1)-C(8)-C(11)$ & $104.1(2)$ \\
\hline$C\left(11^{\prime}\right)-C(8)-C(11)$ & $24.1(3)$ \\
\hline$C(10)-C(8)-C(11)$ & $122.8(3)$ \\
\hline$C(9)-C(8)-C(11)$ & $103.0(3)$ \\
\hline $\mathrm{C}(8)-\mathrm{C}(9)-\mathrm{H}(9 \mathrm{~A})$ & 109.5 \\
\hline $\mathrm{C}(8)-\mathrm{C}(9)-\mathrm{H}(9 \mathrm{~B})$ & 109.5 \\
\hline $\mathrm{H}(9 \mathrm{~A})-\mathrm{C}(9)-\mathrm{H}(9 \mathrm{~B})$ & 109.5 \\
\hline $\mathrm{C}(8)-\mathrm{C}(9)-\mathrm{H}(9 \mathrm{C})$ & 109.5 \\
\hline$H(9 A)-C(9)-H(9 C)$ & 109.5 \\
\hline $\mathrm{H}(9 \mathrm{~B})-\mathrm{C}(9)-\mathrm{H}(9 \mathrm{C})$ & 109.5 \\
\hline $\mathrm{C}(8)-\mathrm{C}(10)-\mathrm{H}(10 \mathrm{~A})$ & 109.5 \\
\hline $\mathrm{C}(8)-\mathrm{C}(10)-\mathrm{H}(10 \mathrm{~B})$ & 109.5 \\
\hline $\mathrm{H}(10 \mathrm{~A})-\mathrm{C}(10)-\mathrm{H}(10 \mathrm{~B})$ & 109.5 \\
\hline$C(8)-C(10)-H(10 C)$ & 109.5 \\
\hline $\mathrm{H}(10 \mathrm{~A})-\mathrm{C}(10)-\mathrm{H}(10 \mathrm{C})$ & 109.5 \\
\hline $\mathrm{H}(10 \mathrm{~B})-\mathrm{C}(10)-\mathrm{H}(10 \mathrm{C})$ & 109.5 \\
\hline$C(8)-C(11)-H(11 A)$ & 109.5 \\
\hline$C(8)-C(11)-H(11 B)$ & 109.5 \\
\hline
\end{tabular}




\begin{tabular}{|c|c|}
\hline $\mathrm{H}(11 \mathrm{~A})-\mathrm{C}(11)-\mathrm{H}(11 \mathrm{~B})$ & 109.5 \\
\hline $\mathrm{C}(8)-\mathrm{C}(11)-\mathrm{H}(11 \mathrm{C})$ & 109.5 \\
\hline $\mathrm{H}(11 \mathrm{~A})-\mathrm{C}(11)-\mathrm{H}(11 \mathrm{C})$ & 109.5 \\
\hline $\mathrm{H}(11 \mathrm{~B})-\mathrm{C}(11)-\mathrm{H}(11 \mathrm{C})$ & 109.5 \\
\hline$C(8)-C\left(11^{\prime}\right)-H(11 D)$ & 109.5 \\
\hline$C(8)-C\left(11^{\prime}\right)-H(11 E)$ & 109.5 \\
\hline $\mathrm{H}(11 \mathrm{D})-\mathrm{C}\left(11^{\prime}\right)-\mathrm{H}(11 \mathrm{E})$ & 109.5 \\
\hline$C(8)-C\left(11^{\prime}\right)-H(11 F)$ & 109.5 \\
\hline $\mathrm{H}(11 \mathrm{D})-\mathrm{C}\left(11^{\prime}\right)-\mathrm{H}(11 \mathrm{~F})$ & 109.5 \\
\hline $\mathrm{H}(11 \mathrm{E})-\mathrm{C}\left(11^{\prime}\right)-\mathrm{H}(11 \mathrm{~F})$ & 109.5 \\
\hline$C(13)-C(12)-P(1)$ & $114.57(9)$ \\
\hline$C(13)-C(12)-H(12 A)$ & 108.6 \\
\hline$P(1)-C(12)-H(12 A)$ & 108.6 \\
\hline$C(13)-C(12)-H(12 B)$ & 108.6 \\
\hline$P(1)-C(12)-H(12 B)$ & 108.6 \\
\hline $\mathrm{H}(12 \mathrm{~A})-\mathrm{C}(12)-\mathrm{H}(12 \mathrm{~B})$ & 107.6 \\
\hline$C(12)-C(13)-H(13 A)$ & 109.5 \\
\hline $\mathrm{C}(12)-\mathrm{C}(13)-\mathrm{H}(13 \mathrm{~B})$ & 109.5 \\
\hline$H(13 A)-C(13)-H(13 B)$ & 109.5 \\
\hline $\mathrm{C}(12)-\mathrm{C}(13)-\mathrm{H}(13 \mathrm{C})$ & 109.5 \\
\hline $\mathrm{H}(13 \mathrm{~A})-\mathrm{C}(13)-\mathrm{H}(13 \mathrm{C})$ & 109.5 \\
\hline $\mathrm{H}(13 \mathrm{~B})-\mathrm{C}(13)-\mathrm{H}(13 \mathrm{C})$ & 109.5 \\
\hline$C(15)-C(14)-P(1)$ & $113.27(9)$ \\
\hline$C(15)-C(14)-H(14 A)$ & 108.9 \\
\hline$P(1)-C(14)-H(14 A)$ & 108.9 \\
\hline$C(15)-C(14)-H(14 B)$ & 108.9 \\
\hline$P(1)-C(14)-H(14 B)$ & 108.9 \\
\hline $\mathrm{H}(14 \mathrm{~A})-\mathrm{C}(14)-\mathrm{H}(14 \mathrm{~B})$ & 107.7 \\
\hline$C(14)-C(15)-H(15 A)$ & 109.5 \\
\hline$C(14)-C(15)-H(15 B)$ & 109.5 \\
\hline $\mathrm{H}(15 \mathrm{~A})-\mathrm{C}(15)-\mathrm{H}(15 \mathrm{~B})$ & 109.5 \\
\hline $\mathrm{C}(14)-\mathrm{C}(15)-\mathrm{H}(15 \mathrm{C})$ & 109.5 \\
\hline $\mathrm{H}(15 \mathrm{~A})-\mathrm{C}(15)-\mathrm{H}(15 \mathrm{C})$ & 109.5 \\
\hline $\mathrm{H}(15 \mathrm{~B})-\mathrm{C}(15)-\mathrm{H}(15 \mathrm{C})$ & 109.5 \\
\hline$C(17)-C(16)-P(1)$ & $116.45(9)$ \\
\hline$C(17)-C(16)-H(16 A)$ & 108.2 \\
\hline
\end{tabular}


(C) 2006 American Chemical Society, Organometallics, Bleeke om058058f Supporting Info Page 103

$\begin{array}{ll}\mathrm{P}(1)-\mathrm{C}(16)-\mathrm{H}(16 \mathrm{~A}) & 108.2 \\ \mathrm{C}(17)-\mathrm{C}(16)-\mathrm{H}(16 \mathrm{~B}) & 108.2 \\ \mathrm{P}(1)-\mathrm{C}(16)-\mathrm{H}(16 \mathrm{~B}) & 108.2 \\ \mathrm{H}(16 \mathrm{~A})-\mathrm{C}(16)-\mathrm{H}(16 \mathrm{~B}) & 107.3 \\ \mathrm{C}(16)-\mathrm{C}(17)-\mathrm{H}(17 \mathrm{~A}) & 109.5 \\ \mathrm{C}(16)-\mathrm{C}(17)-\mathrm{H}(17 \mathrm{~B}) & 109.5 \\ \mathrm{H}(17 \mathrm{~A})-\mathrm{C}(17)-\mathrm{H}(17 \mathrm{~B}) & 109.5 \\ \mathrm{C}(16)-\mathrm{C}(17)-\mathrm{H}(17 \mathrm{C}) & 109.5 \\ \mathrm{H}(17 \mathrm{~A})-\mathrm{C}(17)-\mathrm{H}(17 \mathrm{C}) & 109.5 \\ \mathrm{H}(17 \mathrm{~B})-\mathrm{C}(17)-\mathrm{H}(17 \mathrm{C}) & 109.5 \\ \end{array}$

Symmetry transformations used to generate equivalent atoms:

$\# 1-x,-y+1,-z$ 
(C) 2006 American Chemical Society, Organometallics, Bleeke om058058f Supporting Info Page 104

Table 4. Anisotropic displacement parameters $\left(\AA^{2} \times 10^{3}\right)$ for jb13905. The anisotropic

displacement factor exponent takes the form: $-2 \pi^{2}\left[h^{2} a^{* 2} U^{11}+\ldots+2 h k a^{*} b^{*} U^{12}\right]$

\begin{tabular}{|c|c|c|c|c|c|c|}
\hline & $\mathrm{U}^{11}$ & $\mathrm{U}^{22}$ & $\mathrm{U}^{33}$ & $\mathrm{U}^{23}$ & $U^{13}$ & $\mathrm{U}^{12}$ \\
\hline $\mathrm{Rh}(1)$ & $9(1)$ & $9(1)$ & $11(1)$ & $0(1)$ & $-1(1)$ & $0(1)$ \\
\hline$S(1)$ & $12(1)$ & $11(1)$ & $12(1)$ & $-2(1)$ & $-3(1)$ & $1(1)$ \\
\hline$P(1)$ & $11(1)$ & $11(1)$ & $12(1)$ & $0(1)$ & $-2(1)$ & $O(1)$ \\
\hline$N(1)$ & $18(1)$ & $21(1)$ & $19(1)$ & $4(1)$ & $-1(1)$ & $-3(1)$ \\
\hline$C(1)$ & $13(1)$ & $17(1)$ & $16(1)$ & $-2(1)$ & $2(1)$ & $2(1)$ \\
\hline$C(2)$ & $18(1)$ & $15(1)$ & $18(1)$ & $-4(1)$ & $-3(1)$ & $4(1)$ \\
\hline$C(3)$ & $17(1)$ & $11(1)$ & $15(1)$ & $-2(1)$ & $-5(1)$ & $2(1)$ \\
\hline$C(4)$ & $13(1)$ & $11(1)$ & $13(1)$ & $0(1)$ & $-1(1)$ & $0(1)$ \\
\hline$C(5)$ & $21(1)$ & $21(1)$ & $37(1)$ & $-7(1)$ & $0(1)$ & $8(1)$ \\
\hline$C(6)$ & $22(1)$ & $12(1)$ & $26(1)$ & $0(1)$ & $-9(1)$ & $-1(1)$ \\
\hline$C(7)$ & $14(1)$ & $16(1)$ & $14(1)$ & $0(1)$ & $-2(1)$ & $-1(1)$ \\
\hline$C(8)$ & $26(1)$ & $32(1)$ & $32(1)$ & $20(1)$ & $-1(1)$ & $-9(1)$ \\
\hline$C(9)$ & $53(1)$ & $26(1)$ & $33(1)$ & $11(1)$ & $-1(1)$ & $3(1)$ \\
\hline$C(10)$ & $67(2)$ & $82(2)$ & $53(1)$ & $43(1)$ & $41(1)$ & $39(1)$ \\
\hline$C(11)$ & $33(3)$ & $29(2)$ & $53(4)$ & $12(2)$ & $-8(2)$ & $-19(2)$ \\
\hline$C\left(11^{\prime}\right)$ & $38(3)$ & $55(4)$ & $52(4)$ & $16(3)$ & $-3(2)$ & $-31(3)$ \\
\hline$C(12)$ & $18(1)$ & $15(1)$ & $20(1)$ & $-1(1)$ & $-4(1)$ & $-2(1)$ \\
\hline$C(13)$ & $14(1)$ & $22(1)$ & $36(1)$ & $2(1)$ & $-1(1)$ & $-5(1)$ \\
\hline$C(14)$ & $18(1)$ & $19(1)$ & $14(1)$ & $0(1)$ & $1(1)$ & $4(1)$ \\
\hline$C(15)$ & $22(1)$ & $25(1)$ & $22(1)$ & $4(1)$ & $8(1)$ & $1(1)$ \\
\hline$C(16)$ & $18(1)$ & $13(1)$ & $17(1)$ & $O(1)$ & $-4(1)$ & $2(1)$ \\
\hline$C(17)$ & $27(1)$ & $19(1)$ & $21(1)$ & $2(1)$ & $-9(1)$ & $5(1)$ \\
\hline
\end{tabular}


(C) 2006 American Chemical Society, Organometallics, Bleeke om058058f Supporting Info Page 105

Table 5. Hydrogen coordinates ( $\left.\times 10^{4}\right)$ and isotropic displacement parameters $\left(\AA^{2} \times 10^{3}\right)$ for jb1 3905.

\begin{tabular}{|c|c|c|c|c|}
\hline & $\mathrm{x}$ & $y$ & z & $\mathrm{U}(\mathrm{eq})$ \\
\hline $\mathrm{H}(1 \mathrm{~A})$ & 2761 & 3947 & -1018 & 18 \\
\hline$H(1 B)$ & 3348 & 3914 & 45 & 18 \\
\hline $\mathrm{H}(4)$ & 41 & 3287 & 838 & 15 \\
\hline $\mathrm{H}(5 \mathrm{~A})$ & 3720 & 1702 & 44 & 39 \\
\hline $\mathrm{H}(5 \mathrm{~B})$ & 3447 & 1891 & -1101 & 39 \\
\hline $\mathrm{H}(5 \mathrm{C})$ & 2783 & 983 & -485 & 39 \\
\hline $\mathrm{H}(6 \mathrm{~A})$ & 220 & 1151 & -287 & 30 \\
\hline $\mathrm{H}(6 \mathrm{~B})$ & 443 & 1197 & 879 & 30 \\
\hline $\mathrm{H}(6 \mathrm{C})$ & 1339 & 675 & 163 & 30 \\
\hline $\mathrm{H}(9 \mathrm{~A})$ & 1657 & 8184 & -2106 & 56 \\
\hline $\mathrm{H}(9 \mathrm{~B})$ & 2557 & 9149 & -2117 & 56 \\
\hline $\mathrm{H}(9 \mathrm{C})$ & 1872 & 8920 & -1138 & 56 \\
\hline $\mathrm{H}(10 \mathrm{~A})$ & 4121 & 6593 & -1984 & 101 \\
\hline $\mathrm{H}(10 \mathrm{~B})$ & 3920 & 7656 & -2675 & 101 \\
\hline $\mathrm{H}(10 \mathrm{C})$ & 3000 & 6717 & -2589 & 101 \\
\hline $\mathrm{H}(11 \mathrm{~A})$ & 3308 & 8757 & -92 & 58 \\
\hline $\mathrm{H}(11 \mathrm{~B})$ & 4152 & 9054 & -947 & 58 \\
\hline $\mathrm{H}(11 \mathrm{C})$ & 4332 & 7963 & -287 & 58 \\
\hline$H(11 D)$ & 3893 & 8578 & -304 & 73 \\
\hline $\mathrm{H}(11 \mathrm{E})$ & 4483 & 8604 & -1353 & 73 \\
\hline$H(11 F)$ & 4527 & 7500 & -691 & 73 \\
\hline $\mathrm{H}(12 \mathrm{~A})$ & 3046 & 6674 & 1713 & 21 \\
\hline $\mathrm{H}(12 \mathrm{~B})$ & 3593 & 5971 & 2589 & 21 \\
\hline $\mathrm{H}(13 \mathrm{~A})$ & 4785 & 5114 & 1495 & 36 \\
\hline$H(13 B)$ & 4865 & 6431 & 1327 & 36 \\
\hline $\mathrm{H}(13 \mathrm{C})$ & 4179 & 5677 & 569 & 36 \\
\hline $\mathrm{H}(14 \mathrm{~A})$ & 1916 & 5196 & 3391 & 20 \\
\hline $\mathrm{H}(14 \mathrm{~B})$ & 1105 & 5890 & 2693 & 20 \\
\hline $\mathrm{H}(15 \mathrm{~A})$ & 84 & 4262 & 2364 & 35 \\
\hline$H(15 B)$ & 149 & 4470 & 3531 & 35 \\
\hline
\end{tabular}


(C) 2006 American Chemical Society, Organometallics, Bleeke om058058f Supporting Info Page 106

\begin{tabular}{lrlll}
$\mathrm{H}(15 \mathrm{C})$ & 909 & 3548 & 3033 & 35 \\
$\mathrm{H}(16 \mathrm{~A})$ & 2500 & 2951 & 2053 & 19 \\
$\mathrm{H}(16 \mathrm{~B})$ & 3607 & 3419 & 1603 & 19 \\
$\mathrm{H}(17 \mathrm{~A})$ & 4097 & 4160 & 3170 & 34 \\
$\mathrm{H}(17 \mathrm{~B})$ & 3912 & 2842 & 3235 & 34 \\
$\mathrm{H}(17 \mathrm{C})$ & 2991 & 3677 & 3619 & 34 \\
\hline
\end{tabular}

